OPEN ACCESS

Edited by:

Paul Croarkin

Mayo Clinic, United States

Reviewed by:

Scott Tyler Aaronson,

Sheppard and Enoch Pratt Hospital,

United States

Tapan Parikh

Ann \& Robert H. Lurie Children's Hospital of Chicago, United States

*Correspondence:

Nicholas T. Trapp

nicholas-trapp@uiowa.edu

Specialty section:

This article was submitted to

Neuroimaging and Stimulation,

a section of the journal

Frontiers in Psychiatry

Received: 01 July 2021 Accepted: 06 August 2021 Published: 27 August 2021

Citation:

Trapp NT and Williams NR (2021) The Future of Training and Practice in Neuromodulation: An Interventional

Psychiatry Perspective.

Front. Psychiatry 12:734487.

doi: 10.3389/fpsyt.2021.734487

\section{The Future of Training and Practice in Neuromodulation: An Interventional Psychiatry Perspective}

\author{
Nicholas T. Trapp ${ }^{1,2 \star}$ and Nolan R. Williams ${ }^{1}$ \\ ${ }^{1}$ Department of Psychiatry and Behavioral Sciences, Stanford University, Stanford, CA, United States, ${ }^{2}$ Department of \\ Psychiatry, University of lowa, lowa City, IA, United States
}

Keywords: interventional psychiatry, neuromodulation, repetitive transcranial magnetic stimulation, electroconvulsive therapy, deep brain stimulation, vagus nerve stimulation, training, fellowship

\section{INTRODUCTION}

Psychiatrists have been proceduralists dating back almost 100 years, nearly to psychiatry's inception as a medical specialty. Electroconvulsive therapy (ECT) was developed as a procedure in the 1930 s and refined over time, with impressive efficacy and response rates (1). However, due to a lack of understanding of the pathophysiology of many psychiatric conditions, combined with disastrous outcomes from premature adoption of other procedural treatments such as transorbital lobotomy (2), procedural options for psychiatric treatment have been limited. In recent years this landscape is changing, again introducing the potential for procedural therapies in neuropsychiatric disease management.

The subspecialty focused on procedure-based psychiatric care, specifically utilization of neurotechnologies to treat psychiatric disorders has become known as "interventional psychiatry." Interventional psychiatry frequently employs treatments under the umbrella of "psychiatric neuromodulation," which we define as the collection of nervous system stimulation therapies focused on modulating dysfunctional brain circuitry for therapeutic benefit, including use of electrical, magnetic, ultrasonic, and photic stimulation (see Table $\mathbf{1}$ for examples). As practically applied, interventional psychiatry also often incorporates procedure-based pharmacologic interventions such as ketamine infusion therapy and psychedelic therapies, which similarly involve a medical intervention applied to a treatment-refractory patient population and require familiarity with procedural consent and monitoring. Here we outline the state of the field, as well as implications for training and the role of the interventional psychiatrist in the treatment team.

\section{THE EXPANSION OF NEUROMODULATION AND ADVENT OF INTERVENTIONAL PSYCHIATRY}

There has been an explosion of technology in recent years capable of interfacing with the nervous system. Much of this technology focuses on treating conditions with some evidence for circuit-based or brain network pathology, such as depression, obsessive-compulsive disorder, chronic pain, movement disorders, substance abuse, and epilepsy seizure propagation (3-8). Modulation of brain regions implicated in these circuits or networks has demonstrated therapeutic efficacy, resulting in a multitude of clinical applications across a range of medical disciplines including psychiatry, neurology, neurosurgery, and pain management (9).

The field of interventional psychiatry was conceptualized in its modern form $<10$ years ago $(10,11)$. Around that time, a whole host of novel procedural treatments and devices were entering the psychiatric clinician's armamentarium (12-14). Vagus nerve stimulation (VNS) was FDA-approved for adjunctive treatment of depression (2005), transcranial magnetic stimulation 
(TMS) and deep TMS were FDA-cleared for treatment-refractory depression (2008 \& 2013), and deep brain stimulation (DBS) received a humanitarian device exemption for obsessivecompulsive disorder (2009). Early evidence of the rapid efficacy of ketamine infusions for treatment-resistant depression was mounting (15) and novel convulsive therapies were in development including magnetic seizure therapy (MST) and FEAST $(16,17)$. However, with these new technologies arose concerns about how to disseminate newfound knowledge and ensure adequate training of clinicians on the procedural aspects of care. Interventional psychiatry arose as a notion designed to unify providers around a common craft, to foster discussions about training requirements and their incorporation into residency programs, and to gather like-minded clinicians to guide and further incorporate neurotechnologies in a rapidly expanding field.

\section{THE STATE OF INTERVENTIONAL PSYCHIATRY TRAINING}

The rapid technological advances in neurotherapeutics over the past few decades have relegated prior psychiatric training standards as inadequate for the successful management and administration of these emerging neurotechnologies in psychiatry. Indeed, prior to the past 10-20 years, a psychiatrist's procedural training likely included exposure to electroconvulsive therapy at best, with residency training competencies focused primarily on "understanding the indications and uses" of ECT (18) with no requirement for procedural exposure. This remains a limitation of many training programs today, perpetuated by updated training milestones which make non-specific recommendations with no requirements for direct exposure or procedural training in interventional techniques $(19,20)$.

The level of training a physician may have prior to offering these therapies is highly variable. For ECT, most institutions or hospital systems have credentialing processes in place to ensure some level of proficiency prior to independent practice; however, there is no national standard. The situation is even less standardized for TMS, where many institutions lack a credentialing process. Procedural training experiences for clinicians may vary from a 1-h training from the device manufacturer to a week-long workshop, a month-long residency elective, or more recently, a year-long fellowship in interventional psychiatry. Although evidence linking more experience to better patient outcomes is limited, data suggests that technical aspects of these procedures, such as reliable TMS treatment targeting, are difficult even for trained technicians. Thus, more experience may lead to better technique, such as more reliable TMS treatment targeting or lower TMS motor thresholds (indicating more accurate treatment dosing) (21). Determining adequacy of training can be challenging, and standardizing this across institutions or regions presents an even greater challenge. As the subspecialty of interventional psychiatry continues to grow, more formalized training requirements may be considered to ensure proficiency of providers for optimal patient safety and treatment outcomes.
TABLE 1 | Proposed competency areas for trans-disciplinary fellowship in interventional psychiatry or psychiatric neuromodulation.

\section{CORE COMPETENCIES:}

\section{Invasive neuromodulation}

- Indications, Evaluation, Procedure, Periprocedural Care (DBS, VNS, RNS, SCS, epCS)

- In depth knowledge of treatment approaches, pharmacologic and psychotherapeutic treatment alternatives, risks/benefits/side effects of each procedure

- Demonstrated skill and knowledge for seeking and obtaining patient consent, answering patient and family questions about treatment options

- Demonstrated proficiency in device management including initial programming, impedance checks, troubleshooting device issues, intraoperative neuropsychiatric assessments of patients during awake DBS procedures

- Knowledge of how and when to interface with neurology, neurosurgery, and device manufacturers

- Troubleshooting treatment failures or lack of response to standard settings

- Communication of expectations pre-procedure, patient education, follow-up care, maintenance, and augmentation strategies

Non-invasive neuromodulation

Modalities: Electrical $=t E S, E C T, T N S, P N S ;$ Magnetic $=T M S, M S T$; Pharmacologic $=$ ketamine, esketamine, psychedelics; Ultrasonic $=$ FUS; Photic = photobiomodulation, gamma light therapy, optogenetics; Haptic = micromotor stimulation

- Indications, Evaluation, Procedure, Periprocedural Care

- In depth knowledge of treatment approaches, pharmacologic and psychotherapeutic treatment alternatives, risks/benefits/side effects of each procedure

- Demonstrated skill and knowledge for seeking and obtaining patient consent, answering patient and family questions about treatment options

- Demonstrated skill in administration of each treatment modality, troubleshooting issues and managing side effects and complications

- Troubleshooting treatment failures and lack of response to standard protocols or settings

- Skill in procedure-specific techniques such as reading/interpreting EEG in ECT treatments, achieving motor threshold in TMS procedures, delivering provocation in TMS for OCD

- Knowledge of techniques currently used off-label or without an FDAapproved indication, including potential research applications and future clinical potential

- Communication of expectations pre-procedure, patient education, follow-up care, maintenance and augmentation strategies

\section{Neuroimaging-diagnostic and functional}

- Demonstrated proficiency in localization of neuroanatomical structures and white matter tracts, especially those of relevance for neuromodulation targeting and therapy

Neuropsychiatric evaluations with a focus on treatment refractory OCD, depression, and other conditions amenable to neuromodulation intervention

- Knowledge of common psychiatric symptom rating scales to quantify disease characteristics and treatment response

- Knowledge of proposed neurocircuitry involved in neuropsychiatric diseases and proposed therapeutic options

Cross-disciplinary neuropsychiatric evaluations for patients with conditions amenable to neuromodulation intervention

- Knowledge of indications/contraindications for neuromodulation, necessary work-up and evaluation, treatment alternatives, differential diagnostic considerations

\section{Subspecialty evaluations may include}

- Movement Disorder Evaluations with a focus on Tourette's, Parkinson's Disease, Essential Tremor, Dystonia 
TABLE 1 | Continued

Epilepsy Evaluations, Epilepsy Monitoring Unit Patient Management with a focus on reading EEG or qEEG, cortical mapping procedures in EMU

Pain Evaluations

Neurorehabilitation Evaluations

Neurosurgical Evaluations with a focus on medical contraindications to surgical intervention, surgical approaches and ability to weigh risks and benefits of different surgical interventions, necessary preoperative and perioperative work-up and management, post-operative care and device/wound management

Professionalism and communication skills especially with multidisciplinary care and interfacing with other specialties

Active learning demonstrated by contributions to the field such as scientific publication, quality improvement project, reading and interpreting new relevant journal articles

Cross-disciplinary fellowship options with potential for disease-specific procedural focus

- Psychiatry track with emphasis on TMS, ECT, VNS, ketamine

Neurology track emphasizing DBS, FUS, VNS

- Pain management track emphasizing SCS, tES, TMS, ketamine

- Neurorehab/PM\&R track emphasizing TMS, intrathecal baclofen, SCS

DBS, deep brain stimulation; VNS, vagus nerve stimulation; RNS, responsive neurostimulation; SCS, spinal cord stimulation; epCS, epidural cortical stimulation; tES, transcranial electrical stimulation; ECT, electroconvulsive therapy; TNS, trigeminal nerve stimulation; PNS, peripheral nerve stimulation; TMS, transcranial magnetic stimulation; MST, magnetic seizure therapy; FUS, focused ultrasound.

\section{SHOULD INTERVENTIONAL PSYCHIATRY REQUIRE FELLOWSHIP TRAINING?}

One common method to ensure adequate training and competency amongst physicians within a clinical discipline is the development of a formalized fellowship training program. Fellowships have the benefit of regulating training and monitoring a trainee's completion of various milestones or competencies, with the detriment of limiting clinician access to certain practice privileges in some scenarios, and thus limiting patient access to care. Informal or non-accredited fellowships can provide similar training experiences, but without the same standardization or regulation. Both formal and informal fellowships carry the drawback of requiring clinicians to spend additional time after residency engaged in training, thus further delaying their ability to enter independent practice.

An alternative to fellowship training would be incorporating interventional psychiatry training into residency programs (11). Although ideal in many respects, there are also many limitations. Many psychiatry training programs do not have access to interventional psychiatry equipment, technology, and expertise. Indeed, ECT is the most long-standing neuromodulation technique employed in psychiatry, and yet it lacks a foothold in many training programs. One recent study showed that only $75 \%$ of psychiatry residency training programs required residents to have clinical exposure to ECT, and only $57 \%$ had a dedicated ECT rotation (22). In addition to a lack of access, there is also a lack of time. Residents have numerous demands on their time and an ever-increasing list of competencies and learning expectations; a "sampling" of neuromodulation may be a useful "taster," but is unlikely to allow most residents to achieve proficiency unless they set aside elective time for the endeavor.

Based on the current state of the field, the requirement of a fellowship may be premature but is certainly looming. Numerous fellowships now exist around the country. Most are focused on interventional psychiatry and thus limited to psychiatrists; however, an intriguing development has been the creation of cross-disciplinary "neuromodulation" fellowships open to physicians from diverse clinical training backgrounds including psychiatry, neurosurgery, neurology, and physical medicine and rehabilitation (PM\&R) (23). This crossdisciplinary training approach may have some advantages, such as promoting new ideas and applications for available neurotechnologies or fostering multidisciplinary research and clinical collaborations. Indeed, cross-disciplinary fellowship training mirrors a trend in subspecialties such as interventional neuroradiology/endovascular surgery, where clinicians from various training backgrounds can receive fellowship training in a common field of interest $(24,25)$. These parallel procedural, hightech subspecialty training programs can serve as templates for how to consider restructuring interventional psychiatry training over time. The necessity of formalized fellowship training in interventional psychiatry may be dependent on the evolving scope of practice within this field, discussed below.

\section{WHAT IS AN INTERVENTIONAL PSYCHIATRIST'S SCOPE OF PRACTICE?}

The short answer to this question is "whatever they were trained and/or credentialed to do." Common examples include the administration of ECT, TMS, or ketamine/esketamine, the periprocedural evaluation and management of patients with treatment refractory psychiatric illnesses, and the programming and management of DBS and VNS devices for psychiatric indications. However, significant speculation toward the future can be offered. Often procedural specialties have an evolving scope of practice based on new technologies and new clinical needs. For example, the development of novel endovascular neurosurgical tools and techniques for aneurysm coiling, carotid artery stenting, and clot retrieval has drawn representatives from multiple specialties, including traditionally non-surgical specialties such as neurology and radiology, to seek interventional fellowship training, especially as the clinical demand and infrastructure has grown. Likewise, pain management clinicians with backgrounds in anesthesiology, neurology, PM\&R, emergency medicine, and psychiatry have trained in invasive neuromodulation techniques to manage pain conditions. Scope of practice is thus adapted through the vehicle of fellowship training, expanded residency training, or medical education workshops to teach clinicians to master and incorporate new technologies and skills that are not customarily part of any specific residency training program. In the pain management example above, a board-certified psychiatrist can therefore complete a pain medicine fellowship to themselves implant spinal cord neurostimulators for pain, subsequently deemed within their scope of practice. 
Interventional psychiatrists similarly need to remain poised to adopt, master and incorporate new technologies and treatment modalities as they arise. Potential areas of future practice expansion include incorporation of new non-invasive technologies such as MST, transcranial electrical stimulation, focused ultrasound and trigeminal nerve stimulation. Implantation and management of both emerging and established invasive technologies for psychiatric disease may also be within the scope of interventional psychiatry practice in the future. This could include implanting VNS, DBS, responsive neurostimulation devices, or epidural cortical stimulation devices for depression and other neuropsychiatric conditions. Traditionally, neurosurgeons have performed the more invasive neuromodulation procedures due to the higher degree of skill required and the higher safety risk, thus the necessity of a steady caseload to keep up one's technique. However, as these technologies obtain new indications for highly prevalent psychiatric conditions such as major depressive disorder (lifetime prevalence 20.6\%) (26) and obsessive compulsive disorder (lifetime prevalence 2.3\%) (27), there will likely be increased demand for these neuromodulation procedures and well-trained clinicians to provide them. Interventional modalities often have superior safety and cost-effectiveness profiles (28-34) in treating medication-refractory patients, leading clinicians to call for their use earlier in a disease course $(35,36)$ and further increasing clinical demand.

Current interventional psychiatry fellowship does not train psychiatrists in stereotactic and functional neurosurgical techniques such as device implantation. Were interventional psychiatrists motivated to pursue additional training in these invasive, higher-risk procedures, hybrid invasive/non-invasive training programs could emerge. At that stage, interventional psychiatry fellowship training would become a necessity likely requiring two or more years duration, akin to interventional neurology fellowships designed to teach neurologists to perform intravascular procedures. This longer training would ensure adequate procedural skill for achieving successful and safe patient outcomes.

Based on the current state of the field and the rapid advancement of treatment techniques, we recommend that, at

\section{REFERENCES}

1. Sackeim HA. Modern electroconvulsive therapy: vastly improved yet greatly underused. JAMA Psychiatry. (2017) 74:77980. doi: 10.1001/jamapsychiatry.2017.1670

2. Green JR, Duisberg RE, McGrath WB. Orbitofrontal lobotomy with reference to effects on 55 psychotic patients. J Neurosurg. (1952) 9:57987. doi: 10.3171/jns.1952.9.6.0579

3. Padmanabhan JL, Cooke D, Joutsa J, Siddiqi SH, Ferguson M, Darby RR, et al. A Human depression circuit derived from focal brain lesions. Biol Psychiatry. (2019) 86:749-58. doi: 10.1016/j.biopsych.2019.07.023

4. Maia TV, Cooney RE, Peterson BS. The neural bases of obsessivecompulsive disorder in children and adults. Dev Psychopathol. (2008) 20:1251-83. doi: 10.1017/S0954579408000606

5. Caston RM, Smith EH, Davis TS, Rolston JD. The cerebral localization of pain: anatomical and functional considerations for targeted electrical therapies. $J$ Clin Med. (2020) 9:1945. doi: 10.3390/jcm9061945 a minimum, interventional psychiatry practitioners complete a 1-month interventional psychiatry rotation during residency training or hands-on continuing education training course in the modality of interest prior to its implementation and use. Details of minimum number of treatments to achieve proficiency, regulatory bodies to enforce requirements, grandfathering of current practitioners, and continuing medical education requirements within the expanding subspecialty are all important topics for future discussion. An outline of potential competency areas for a psychiatric neuromodulation or interventional psychiatry fellowship is provided in Table $\mathbf{1}$.

\section{DISCUSSION}

Neuromodulation is a discipline focused on the application of various technologies to the nervous system to effect therapeutic change. It has clinical utility in several branches of medicine including psychiatry, neurology, neurosurgery, pain management, and $P M \& R$. Interventional psychiatry is the subspecialty "home" for providers with experience in psychiatric neuromodulation or other procedural techniques. As has been the case for neuromodulation subspecialties in other areas of medicine, interventional psychiatry is rapidly evolving, both in terms of scope of practice and training needs. This article addresses some important looming questions for the field to consider as leaders and educators contemplate how to adequately train and define the role of interventional psychiatrists. Interventional psychiatry is one of the most exciting, rapidly growing, and promising fields of medicine; responsibly ushering in new therapeutics to providers with the clinical acumen and skill to administer them safely and effectively is a critical step toward improving patient health, function, and well-being.

\section{AUTHOR CONTRIBUTIONS}

NT was involved in the conceptualization of the work and writing the original draft of the manuscript. NW was involved in conceptualization and editing of the manuscript. Both authors contributed to the article and approved the submitted version.
6. Albin RL, Young AB, Penney JB. The functional anatomy of basal ganglia disorders. Trends Neurosci. (1989) 12:36675. doi: 10.1016/0166-2236(89)90074-X

7. Ersche KD, Meng C, Ziauddeen H, Stochl J, Williams GB, Bullmore ET, et al. Brain networks underlying vulnerability and resilience to drug addiction. Proc Natl Acad Sci USA. (2020) 117:15253-61. doi: 10.1073/pnas.20025 09117

8. Bouwens van der Vlis TAM, Schijns O, Schaper F, Hoogland G, Kubben P, Wagner L, et al. Deep brain stimulation of the anterior nucleus of the thalamus for drug-resistant epilepsy. Neurosurg Rev. (2019) 42:28796. doi: 10.1007/s10143-017-0941-x

9. Society IN. Neuromodulation Therapies - Patient Information. San Francisco, CA (2020). Available online at: https://www.neuromodulation.com/therapiespatient (accessed: November 24, 2020)

10. Williams NR, Taylor JJ, Kerns S, Short EB, Kantor EM, George MS. Interventional psychiatry: why now? J Clin Psychiatry. (2014) 75:8957. doi: 10.4088/JCP.13108745 
11. Williams NR, Taylor JJ, Snipes JM, Short EB, Kantor EM, George MS. Interventional psychiatry: how should psychiatric educators incorporate neuromodulation into training? Acad Psychiatry. (2014) 38:168-76. doi: 10.1007/s40596-014-0050-x

12. Lefaucheur JP, Aleman A, Baeken C, Benninger DH, Brunelin J, Di Lazzaro $\mathrm{V}$, et al. Evidence-based guidelines on the therapeutic use of repetitive transcranial magnetic stimulation (rTMS): an update 2014-2018. Clin Neurophysiol. (2020) 131:474-528. doi: 10.1016/j.clinph.2020.02.003

13. Lozano AM, Lipsman N, Bergman H, Brown P, Chabardes S, Chang JW, et al. Deep brain stimulation: current challenges and future directions. Nat Rev Neurol. (2019) 15:148-60. doi: 10.1038/s41582-018-0128-2

14. Johnson RL, Wilson CG. A review of vagus nerve stimulation as a therapeutic intervention. J Inflamm Res. (2018) 11:203-13. doi: 10.2147/JIR.S163248

15. Berman RM, Cappiello A, Anand A, Oren DA, Heninger GR, Charney DS, et al. Antidepressant effects of ketamine in depressed patients. Biol Psychiatry. (2000) 47:351-4. doi: 10.1016/S0006-3223(99)00230-9

16. Sahlem GL, Short EB, Kerns S, Snipes J, DeVries W, Fox JB, et al. Expanded safety and efficacy data for a new method of performing electroconvulsive therapy: focal electrically administered seizure therapy. J ECT. (2016) 32:197203. doi: 10.1097/YCT.0000000000000328

17. Kayser S, Bewernick BH, Grubert C, Hadrysiewicz BL, Axmacher N, Schlaepfer TE. Antidepressant effects, of magnetic seizure therapy and electroconvulsive therapy, in treatment-resistant depression. J Psychiatr Res. (2011) 45:569-76. doi: 10.1016/j.jpsychires.2010.09.008

18. Dolenc TJ, Philbrick KL. Achieving competency in electroconvulsive therapy: a model curriculum. Acad Psychiatry. (2007) 31:657. doi: 10.1176/appi.ap.31.1.65

19. Kinzie JM, DeJong SM, Edgar L, Houston LJ, Janssen F, Macaluso M, et al. Psychiatry milestones 2.0: using the supplemental guide to create a shared model of the development of professional identity and expertise. Acad Psychiatry. (2021) 45:500-5. doi: 10.1007/s40596-021-01455-6

20. Macaluso M, Kinzie M, Cowley D, Houston LJ, DeJong S, Janssen F, et al. The psychiatry milestones 2.0: how did we get from 1.0 to 2.0 and what can users expect? Acad Psychiatry. (2020) 44:779-84. doi: 10.1007/s40596-020-0 1275-0

21. Trapp NT, Bruss J, King Johnson M, Uitermarkt BD, Garrett L, Heinzerling A, et al. Reliability of targeting methods in TMS for depression: beam F3 vs. 5.5 cm. Brain Stimul. (2020) 13:578-81. doi: 10.1016/j.brs.2020.01.010

22. Dinwiddie SH, Spitz D. Resident education in electroconvulsive therapy. $J$ ECT. (2010) 26:310-6. doi: 10.1097/YCT.0b013e3181cb5f78

23. Neuromodulation Medicine Fellowship. University of Minnesota. (2021). Available online at: https://med.umn.edu/psychiatry/ education-training/fellowships/neuromodulation-medicine (accessed June 23, 2021).

24. Higashida RT. Evolution of a new multidisciplinary subspecialty: interventional neuroradiology/neuroendovascular surgery. AJNR Am J Neuroradiol. (2000) 21:1151-2.

25. Wholey MH, Wholey M, Bergeron P, Diethrich EB, Henry M, Laborde JC, et al. Current global status of carotid artery stent placement. Cathet Cardiovasc Diagn. (1998) 44:1-6. doi: 10.1002/(sici)1097-0304(199805)44:1<1::aidccd1 $>3.0$. co;2-b

26. Hasin DS, Sarvet al, Meyers JL, Saha TD, Ruan WJ, Stohl M, et al. Epidemiology of adult DSM-5 major depressive disorder and its specifiers in the United States. JAMA Psychiatry. (2018) 75:336-46. doi: 10.1001/jamapsychiatry.2017.4602
27. Ruscio AM, Stein DJ, Chiu WT, Kessler RC. The epidemiology of obsessivecompulsive disorder in the national comorbidity survey replication. Mol Psychiatry. (2010) 15:53-63. doi: 10.1038/mp.2008.94

28. Simpson KN, Welch MJ, Kozel FA, Demitrack MA, Nahas Z. Costeffectiveness of transcranial magnetic stimulation in the treatment of major depression: a health economics analysis. Adv Ther. (2009) 26:34668. doi: 10.1007/s12325-009-0013-x

29. Ross EL, Zivin K, Maixner DF. Cost-effectiveness of electroconvulsive therapy vs pharmacotherapy/psychotherapy for treatmentresistant depression in the United States. JAMA Psychiatry. (2018) 75:713-22. doi: 10.1001/jamapsychiatry.2018.0768

30. Sperling W, Reulbach U, Kornhuber J. Clinical benefits and cost effectiveness of vagus nerve stimulation in a long-term treatment of patients with major depression. Pharmacopsychiatry. (2009) 42:85-8. doi: 10.1055/s-0028-1103294

31. Pietzsch JB, Garner AM, Marks WJ Jr. Cost-effectiveness of deep brain stimulation for advanced parkinson's disease in the United States. Neuromodulation. (2016) 19:689-97. doi: 10.1111/ner.12474

32. Dams J, Siebert U, Bornschein B, Volkmann J, Deuschl G, Oertel WH, et al. Cost-effectiveness of deep brain stimulation in patients with Parkinson's disease. Mov Disord. (2013) 28:763-71. doi: 10.1002/mds.25407

33. Rossi S, Antal A, Bestmann S, Bikson M, Brewer C, Brockmoller J, et al. Safety and recommendations for TMS use in healthy subjects and patient populations, with updates on training, ethical and regulatory issues: expert guidelines. Clin Neurophysiol. (2021) 132:269-306. doi: 10.1016/j.clinph.2020.10.003

34. Rossi S, Hallett M, Rossini PM, Pascual-Leone A, Safety of TMSCG. Safety, ethical considerations, and application guidelines for the use of transcranial magnetic stimulation in clinical practice and research. Clin Neurophysiol. (2009) 120:2008-39. doi: 10.1016/j.clinph.2009.08.016

35. Hacker ML, Turchan M, Heusinkveld LE, Currie AD, Millan $\mathrm{SH}$, Molinari AL, et al. Deep brain stimulation in earlystage Parkinson disease: five-year outcomes. Neurology. (2020) 95:e393-401. doi: 10.1212/WNL.0000000000009946

36. Conway CR, George MS, Sackeim HA. Toward an evidence-based, operational definition of treatment-resistant depression: when enough is enough. JAMA Psychiatry. (2017) 74:9-10. doi: 10.1001/jamapsychiatry.2016.2586

Conflict of Interest: The authors declare that the research was conducted in the absence of any commercial or financial relationships that could be construed as a potential conflict of interest.

Publisher's Note: All claims expressed in this article are solely those of the authors and do not necessarily represent those of their affiliated organizations, or those of the publisher, the editors and the reviewers. Any product that may be evaluated in this article, or claim that may be made by its manufacturer, is not guaranteed or endorsed by the publisher.

Copyright (c) 2021 Trapp and Williams. This is an open-access article distributed under the terms of the Creative Commons Attribution License (CC BY). The use, distribution or reproduction in other forums is permitted, provided the original author(s) and the copyright owner(s) are credited and that the original publication in this journal is cited, in accordance with accepted academic practice. No use, distribution or reproduction is permitted which does not comply with these terms. 